\title{
CALIDAD DE VIDA EN CÁNCER DE MAMA METASTÁSICO DE LARGA EVOLUCIÓN: LA OPINIÓN DE LAS PACIENTES
}

\author{
LONG-TERM METASTATIC BREAST CANCER PATIENT'S QUALITY OF LIFE: PATIENT'S \\ OPINION
}

\author{
Catalina Falo Zamora', Helena Villar Abelló2, Eva Rodríguez Bruzos², Marisa Mena Cervignon ${ }^{1}$ \\ y Antoni Font Guiteras ${ }^{2}$
}

I Institut Català d'Oncologia L'Hospitalet de Llobregat, Barcelona.

2 Universitat Autònoma de Barcelona. Barcelona.

\section{Resumen}

La supervivencia de las pacientes diagnosticadas de cáncer de mama metástasico ha aumentado en los últimos años gracias a la mejora en los tratamientos. Con todo hay pocos estudios que evalúen la calidad de vida en esta situación. El objetivo de nuestro estudio es mejorar el conocimiento sobre la calidad de vida de estas pacientes complementando la información obtenida en los cuestionarios de calidad de vida existentes con una entrevista de preguntas abiertas. Material y métodos: entre junio de 2011 y febrero 2012 se realizaron un total de 24 entrevistas con un cuestionario basado en preguntas de carácter médico y de carácter psicosocial. Resultados: un $58 \%$ de las pacientes opinan que el tratamiento mejora la calidad de vida y un $83,3 \%$ que los efectos secundarios compensan frente al beneficio de la quimioterapia; las exploraciones complementarias les dan seguridad. Únicamente dos tercios de las pacientes manifiestan tener apoyo social y un tercio no comparte el malestar con nadie. Los aspectos de la enfermedad que más les cuesta afrontar son en orden de importancia: la familia, la pérdida de autonomía, el miedo a la incertidumbre y la progresión de la enfermedad. La mitad de las pacientes opinan que la enfermedad ha aportado un valor positivo en sus vidas. Conclusión: la actitud de las

\section{Abstract}

Metastasic breast cancer patients live longer thanks to the lasts improvements in their treatment. Nonetheless, there exist few studies focused on the quality of live (QoL) of those patients. The aim of our study is to improve the knowledge of metastatic breast cancer patient's quality of life, for this purpose we have elaborated an open questionnaire as a complement to the QoL tests available. Material and methods: From June 2011 to February 2012 we conducted 24 interviews with a questionnaire based on medical and psychosocial items. Results: $58 \%$ of patients considered that treatment improved their QoL, an 83.3\% expressed that the benefit of the treatment compensated for the secondary effects, complementary tests give them relieve, control over the illness and information. Only two thirds of those patients expressed to have social support, and one third said to share their grief with nobody. The main concerns expressed by the patients were in order of value: their family, loss of self-caring, fear about the future and to the illness progression. Half of the patients think that the disease has brought some value to their lives such as improving their scale of values and to improve empathy to somebody else's problems. Conclusion: Metastatic breast cancer patient's attitude is

Correspondencia:

Dra. Catalina Falo Zamora

Servicio de Oncología Médica y Unidad Funcional de Mama.

Institut Català d’Oncologia. IDIBELL.

Avinguda Gran Via de L'Hospitalet 199-203, 08908-L'Hospitalet de Llobregat (Barcelona)

E-mail: cfalo@iconcologia.net 
pacientes metastásicas de larga evolución es admirable, por lo bien adaptadas que están a la situación. No obstante, recomendamos poder ofrecerles el apoyo y la información que necesitan para compensar la sensación de soledad frente a la enfermedad.

Palabras clave: Calidad de vida, pacientes con cáncer de mama metastásico larga evolución, adaptación, información y soporte. admirable in the way they are adapted to such a wearing situation. Nonetheless it's imperative to offer them the help and the medical information they need to compensate for their solitude in front of the illness.

Key words: Quality of life, long-term metastatic breast cancer patients, adaptation, information and support.

\section{INTRODUCCIÓN}

La neoplasia de mama es la primera causa de muerte por cáncer en la mujer de los países desarrollados ${ }^{(1)}$.

A pesar de los notables avances en la supervivencia, gracias a las importantes mejoras acaecidas en el diagnóstico y en los tratamientos, la supervivencia global ha alcanzado el $82,8 \%$ a los 5 años ${ }^{(2)}$, pero todavía una quinta parte de las pacientes se enfrentan a un diagnóstico de enfermedad metastásica.

Mientras que las pacientes recién diagnosticadas de cáncer de mama presentan con frecuencia síntomas de miedo, e incertidumbre sobre el futuro, la mayoría de estos síntomas disminuyen a medida que pasa el tiempo y mejoran las perspectivas de la paciente ${ }^{(3)}$. En cambio, las pacientes que se enfrentan a un diagnóstico de cáncer avanzado presentan mayor número de síntomas físicos y emocionales, con el agravante de que las expectativas de futuro empeoran con el tiempo. Con frecuencia se observa una disminución progresiva de la calidad de vida, y los tratamientos no pueden evitar el empeoramiento físico, el aislamiento social y finalmente la muerte ${ }^{(4)}$.

El pronóstico de las pacientes con cáncer de mama metastásico ha mejorado en los últimos 5-10 años gracias al desarrollo de nuevos fármacos más eficaces y mejor tolerados. Como consecuencia las pacien- tes viven más años, con una supervivencia media del $51 \%$ a los 3 años ${ }^{(5)}$. Con todo, la evolución de cada paciente puede ser muy variable en función de la biología del tumor y la localización de las metástasis. Así las pacientes con tumores triples negativos y afectación visceral la mediana de supervivencia está en torno a los 21 meses, mientras que las pacientes con tumores luminal A y enfermedad únicamente ósea o de partes blandas, puede prolongarse incluso más allá de los 10 años ${ }^{(6)}$. Por este motivo, cada vez más, se considera la enfermedad metastásica como una "enfermedad crónica"(7).

El objetivo del tratamiento en pacientes metastásicas incluye el intento de prolongar la esperanza de vida y a su vez paliar los síntomas de la enfermedad, teniendo en cuenta los efectos secundarios. El tratamiento óptimo de dichas enfermas debería incluir no solo los aspectos técnicos del manejo de la patología, sino considerar también las necesidades de las pacientes en el terreno psicosocial. Es importante ofrecer la información y manifestar una buena comunicación con el equipo médico para mejorar la calidad de vida de las pacientes.

El tiempo que dure la enfermedad las pacientes recibirán tratamiento constante y visitas médicas frecuentes. Los efectos secundarios de los tratamientos se suman a los síntomas propios de la enfermedad como por ejemplo, el dolor, el cansancio y la disnea. A pesar de que 
los oncólogos dedican una gran parte de su actividad a las pacientes metastásicas (aproximadamente el 50\% de las visitas de tratamiento), esta dedicación puede resultar insuficiente. Por un lado, la consulta oncológica está centrada en paliar los efectos secundarios y evaluar la respuesta a los tratamientos, por lo que no suele abarcar otros aspectos también importantes para la paciente como el malestar emocional que comporta la enfermedad. Por otro lado, los cuestionarios de calidad de vida existentes actualmente, a veces tampoco reflejan la situación real de estas pacientes por ser poco específicos respecto a la enfermedad metastásica ${ }^{(8,9)}$. Es fruto de la necesidad de profundizar más en la calidad de vida de estas pacientes, que diseñamos este estudio multidisciplinar.

El objetivo de este estudio multidisciplinar de colaboración oncólogo-psicooncólogo es profundizar en las opiniones de los pacientes respecto a aspectos médicos y psicosociales importantes de su calidad de vida, con el fin de aportar información relevante y complementaria a la que proporcionan las pruebas estandarizadas de calidad de vida.

\section{MATERIAL Y MÉTODOS}

\section{Participantes}

Durante los meses de junio de 2011 a febrero 2012 se seleccionaron de forma consecutiva las pacientes con cáncer de mama con enfermedad metastásica de la agenda de la oncóloga participante en el estudio, para la fase piloto.

Inicialmente se dispuso de una muestra de 45 pacientes diagnosticadas de cáncer de mama metastásico de larga evolución del ICO Hospitalet.

Los criterios de inclusión fueron pacientes diagnosticadas de cáncer de mama metastásico de larga evolución (igual o su- perior a un año), y como criterios de exclusión las pacientes con cáncer de mama metastásico con pocos síntomas de enfermedad que no requieren más de dos visitas al año y aquellas pacientes en que su edad avanzada (> 80 años) dificultara la realización de una entrevista de una hora de duración.

Teniendo en cuenta estos criterios, se han excluido 10 pacientes, 3 por la presentación de pocos síntomas de enfermedad y 7 por edad avanzada. De las 35 pacientes a las que se les ofreció participar en el estudio piloto, no acudieron a la entrevista 5 pacientes por exitus, 2 por circunstancias familiares, 1 por empeoramiento clínico y 3 mujeres rechazaron participar en el estudio, por lo que finalmente se cuenta con una muestra de 24 pacientes con enfermedad metastásica, que representa una participación del $68,57 \%$.

\section{Instrumentos}

Los instrumentos utilizados son la historia clínica y un guión de preguntas abiertas que incluyen las diferentes áreas y aspectos considerados de interés para que las pacientes pudieran expresar libremente sus preocupaciones. Así mismo, también se rellenaron los siguientes instrumentos estandarizados: cuestionario de la EORTC QLQ-C30 ${ }^{(10,11)}$, cuestionario específico para mama EORTC QLQ-BR23 ${ }^{(12)}$ y el cuestionario de calidad de vida QL-CAAFex de Font ${ }^{(13,14)}$. Los resultados de los instrumentos estandarizados serán comunicados en otra publicación pendiente de revisión (E. Rodríguez et al., in press).

\section{Historia clínica}

La historia clínica se ha elaborado específicamente para este estudio e incluye los siguientes apartados: Datos sociodemográficos (edad, estado civil, estudios 
y genograma), datos clínicos (fecha de diagnóstico, tratamiento oncológico previo, fecha de la recidiva, localización de la recidiva y tratamiento oncológico actual, valoración de la respuesta), datos psicológicos (antecedentes personales de trastorno del estado de ánimo, si recibió tratamiento psicológico y/o psicofarmacológico, terapia psicológica en la actualidad, si recibe actualmente tratamiento psicofarmacológico, en caso negativo, si le gustaría recibir, acontecimientos vitales estresantes y observaciones).

\section{Entrevista cualitativa}

Teniendo en cuenta las áreas coincidentes de los cuestionarios anteriormente mencionados, hemos desarrollado un guión de preguntas abiertas, en base a la experiencia previa de los profesionales en el trato con enfermas metastásicas de cáncer de mama a lo largo de los años, para complementar los aspectos considerados y profundizar mediante la opinión de las pacientes. El guión de preguntas abiertas está centrado en aspectos médicos y psicosociales: en primer lugar, los aspectos médicos relacionados con los tratamientos, los efectos secundarios y las pruebas complementarias; en segundo lugar, el apoyo familiar y social, preguntas sobre aspectos emocionales, cognitivos y conductuales como el estilo de afrontamiento, la capacidad funcional para llevar a cabo actividades, las preocupaciones principales de las pacientes y aspectos positivos de la experiencia. Ver la tabla 1.

Esta parte de la entrevista fue grabada con el permiso de las pacientes y transcrita posteriormente por las psicooncólogas, y es el objeto del presente estudio.

\section{Tabla 1. Preguntas abiertas}

\section{Aspectos médicos}

a. El tratamiento:

i. ¿El tratamiento ha mejorado o ha empeorado su calidad de vida?

ii. ¿Qué espera del tratamiento?

iii. ¿Le compensa el beneficio teniendo en cuenta los efectos secundarios?

iv. ¿Qué cree que puede mejorar del tratamiento?

v. ¿Qué tipo de tratamiento querría?

b. Efectos secundarios:

i. ¿Qué efectos secundarios Ileva mejor y cuales peor?

ii. ¿Qué hace para evitar los efectos secundarios en lo posible?

c. Exploraciones complementarias:

i. ¿Piensa que las exploraciones complementarias que le realizan son excesivas?

ii. Las pruebas complementarias le generan angustia o seguridad?

iii. ¿Qué pruebas complementarias cree que empeoran su calidad de vida? ¿Y cuáles la mejoran?

iv. ¿Encuentra a faltar alguna prueba complementaria?

2. Aspectos psicosociales

a. Apoyo sociofamiliar

b. ¿Con quién comparte su malestar emocional?

c. ¿Qué aspectos de la enfermedad le cuesta más afrontar?

d. ¿Qué cosas/actividades disminuyen su malestar emocional?

e. ¿Qué actividades diarias le ocupan más tiempo?

f. ¿A qué querría dedicar más tiempo?

g. ¿Hasta qué punto la enfermedad disminuye su calidad de vida?

h. ¿Qué aspectos de la enfermedad empeoran su calidad de vida?

i. ¿Qué es lo que más le preocupa?

j. ¿Considera que hay algún aspecto positivo o que ha aprendido alguna cosa de la enfermedad?

\section{Procedimiento}

La oncóloga explicaba la existencia 
de este estudio sobre la calidad de vida de las mujeres con cáncer de mama metastásico, y se invitaba a las pacientes a participar. Las pacientes que aceptaban mediante consentimiento informado, eran derivadas a la psicooncóloga, que realizaba las entrevistas abiertas. Las entrevistas fueron llevadas a cabo indistintamente por una de las dos psicooncólogas del estudio, siempre y cuando no fueran pacientes propias para no condicionar las respuestas.

El estudio fue aprobado por el Comité Ético del Hospital Universitario de Bellvitge. En el transcurso de la entrevista, se recogían los datos de la Historia Clínica y posteriormente, se realizaba la valoración de la calidad de vida con el guión de preguntas abiertas.

\section{Análisis estadístico}

El análisis estadístico ha sido realizado con el software: SPSS v14.0 para Windows y SAS v9.1, SAS Institute Inc., Cary, NC, USA.

Este estudio está basado en un análisis descriptivo de los datos. Las entrevistas fueron transcritas de forma fidedigna a un documento Word. Se han recogido las respuestas de las pacientes pregunta por pregunta de forma transversal para poder agrupar las respuestas de las pacientes para cada ítem. Las categorías han sido generadas a partir de las repuestas obtenidas sobre los aspectos médicos y los aspectos psicosociales, y las subcategorías incluidas en estos dos grandes apartados.

\section{RESULTADOS}

\section{Características sociodemográficas de la muestra}

A continuación, se muestran las características sociodemográficas de la muestra. EI grupo está formado por 24 mujeres, la edad media es de 56,04 años con una desviación típica de 9,6, y con edades comprendidas entre 37 y 71 años. La mayor parte de la muestra de pacientes está casada (75\%), tiene hijos $(87,5 \%)$, la mitad de las mujeres tienen estudios primarios $(50 \%)$ y la otra mitad secundarios $(20,8)$ y superiores $(29,2 \%)$.

La edad se ha dividido en cuatro intervalos, y observamos que prácticamente la mitad de la muestra tiene más de 55 años, seguido de las mujeres con edades comprendidas entre 50 y 55 años. Ver la tabla 2.

Tabla 2. Características sociodemográficas de la muestra

\begin{tabular}{c|cc}
\hline $\begin{array}{c}\text { Características socio } \\
\text { demográficas }\end{array}$ & \multicolumn{2}{|c}{$\begin{array}{c}\text { Pacientes } \\
\text { metastásicas } \\
\mathrm{N}=24\end{array}$} \\
\hline & $\mathrm{n}$ & $\%$ \\
\cline { 2 - 3 } Edad (en años) & \multicolumn{2}{c}{$\%$} \\
$\quad$ Mediana años & $56,04(37-71)$ & \\
$<44$ & 3 & 12,5 \\
$44-49$ & 2 & 8,3 \\
$50-55$ & 8 & 33,3 \\
$>55$ & 11 & 45,8
\end{tabular}

Estado civil

$\begin{array}{lcc}\text { Soltera } & 1 & 4,2 \\ \text { Casada } & 18 & 75,0 \\ \text { Divorciada } & 1 & 4,2 \\ \text { Viuda } & 4 & 16,7\end{array}$

Hijos

Sí

No

Estudios

\begin{tabular}{lcc} 
Primarios & 12 & 50,0 \\
Secundarios & 5 & 20,8 \\
Superiores & 7 & 29,2 \\
\hline
\end{tabular}

$\mathrm{N}=$ Número total de participantes $\mathrm{n}=$ número de participante. 
$\%=$ porcentaje de participantes.

\section{Características clínicas}

\section{Estadio}

En tres casos (12,5\%) las pacientes fueron diagnosticadas con enfermedad metastásica al diagnóstico (estadio IV) y el resto se había convertido en metastásicas después de un tiempo libre de enfermedad. La mediana de tiempo libre de enfermedad, definido como el tiempo desde el primer tratamiento recibido con intención radical, hasta la fecha del diagnóstico de enfermedad metastásica ha sido de 5,29 años (rango entre 1,58 y 12,59 años). La mediana de tiempo que las pacientes han convivido con la enfermedad desde el diagnóstico de enfermedad metastásica hasta la fecha de la entrevista ha sido de 3,82 años (rango 1,25 a 15,6 años).

\section{Localización de las metástasis}

La mayoría de las pacientes presentan enfermedad metastásica ósea, pleural, ganglionar y cutánea, agrupadas como metástasis de partes blandas (15 casos). Aunque en 9 casos presentaban enfermedad visceral con afectación hepática, sistema nervioso central, pulmonar, una de ellas incluso con afectación endobronquial confirmada por broncoaspirado (BAS).

\section{Tratamientos recibidos}

Es un grupo de pacientes poli tratadas tanto con varias líneas de quimioterapia (QT) como de hormonoterapia (HT). Ver tabla 3 .

Un $83,3 \%$ de las pacientes habían recibido en algún momento de su enfermedad metastásica un tratamiento con quimioterapia, tanto endovenosa como oral, con una mediana de 2 líneas por paciente (ran- go entre 1 y 6).

Un 70,83\% de las pacientes habían sido tratadas con tratamiento hormonal. La mediana de líneas hormonales por paciente fue de 3 (rango entre 1 y 6 ).

\section{Tabla 3. Características clínicas del grupo metastásico}

\begin{tabular}{lcc}
\hline \multicolumn{1}{c}{$\begin{array}{l}\text { Características } \\
\text { clínicas }\end{array}$} & $\begin{array}{c}\text { Grupo metástasis } \\
\mathrm{N}=24\end{array}$ \\
\hline & $\mathrm{n}$ & $\%$ \\
\cline { 2 - 3 } $\begin{array}{l}\text { Localización } \\
\text { metástasis }\end{array}$ & & \\
$\quad$ Metástasis partes & 15 & 62,5 \\
$\quad$ blandas & & \\
$\quad$ Metátasis viscerales & 9 & 37,5 \\
$\begin{array}{l}\text { Tratamientos recibidos } \\
\text { para enfermedad }\end{array}$ & & \\
metastásica & & \\
$\quad$ QT & 20 & 83,33 \\
HT & 17 & 70,83 \\
$\quad$ Trastuzumab & 3 & 12,50 \\
Bifosfanatos EV & 16 & 66,60
\end{tabular}

Tratamientos oncológicos presentes

Tratamiento hormonal 29,16

$\begin{array}{llc}\text { QMT oral } & 9 & 37,5 \\ \text { QMT endovenosa } & 4 & 16,6 \\ \text { Trastuzumab } & 3 & 12,5 \\ \text { Bifosfanatos EV } & 16 & 66,60 \\ \text { No tratamiento } & 1 & 4\end{array}$

Respuesta al tratamiento

\begin{tabular}{lcc} 
Respuesta & 12 & 50,0 \\
Enfermedad estable & 9 & 37,5 \\
Progresión & 2 & 8,3 \\
No procede & 1 & 4 \\
\hline
\end{tabular}

\section{Opiniones de las pacientes}

Se han Ilevado a cabo 24 entrevistas 
con el guión de preguntas abiertas. La mediana de tiempo de entrevista por paciente fue de una hora (entre 45 y 90 minutos). Las preguntas se agrupaban en dos grandes temas: aspectos médicos y aspectos psicosociales.

\section{Opiniones sobre aspectos médicos}

\section{El tratamiento}

Más de la mitad de las pacientes consideran que el tratamiento ha mejorado su calidad de vida en 15 de los 24 casos $(62,5 \%)$, mientras que 5 pacientes $(20,8 \%)$ no encontraron mejoría con el tratamiento y en 4 casos $(16,6 \%)$ encontraron mejoría en ciertos aspectos pero en otros no. Ver la figura 1 .

Al preguntarles específicamente que esperaban del tratamiento, la mayoría se conformaban con que la enfermedad se detuviera, un porcentaje menor mejorar y en algún caso curarse. Ver la figura 2 .

Según se observa en la tabla 4, aproximadamente la mitad de las pacientes espera que el tratamiento detenga la enfermedad, únicamente un $12,5 \%$ aspira a curarse.

La mayoría de las pacientes consideran que el beneficio del tratamiento compensa el malestar que generan los efectos secundarios (83,3\% de los casos). En la anamnesis se debe distinguir entre efectos secundarios y síntomas derivados de la enfermedad, porque en algún caso hay pacientes que no lo saben diferenciar, por ejemplo, un caso que consideraba su dolor de cabeza derivado del tratamiento, literal: "Ios efectos secundarios me atacan

\section{Figura 1. El tratamiento mejora la calidad de vida en un $\mathbf{6 2 , 5 \%}$ de las pacientes} entrevistadas.

\section{¿El tratamiento mejora la calidad de Vida?}

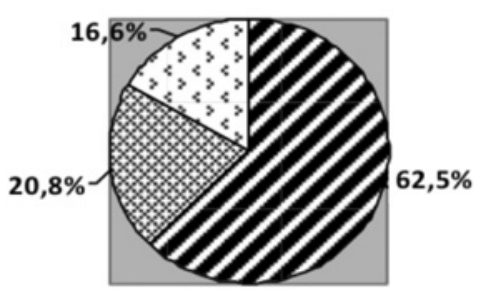

$$
\begin{aligned}
& \text { 口Si } \\
& \text { QNo } \\
& \text { En algun aspecto }
\end{aligned}
$$

Figura 2. ¿Qué espera del tratamiento?

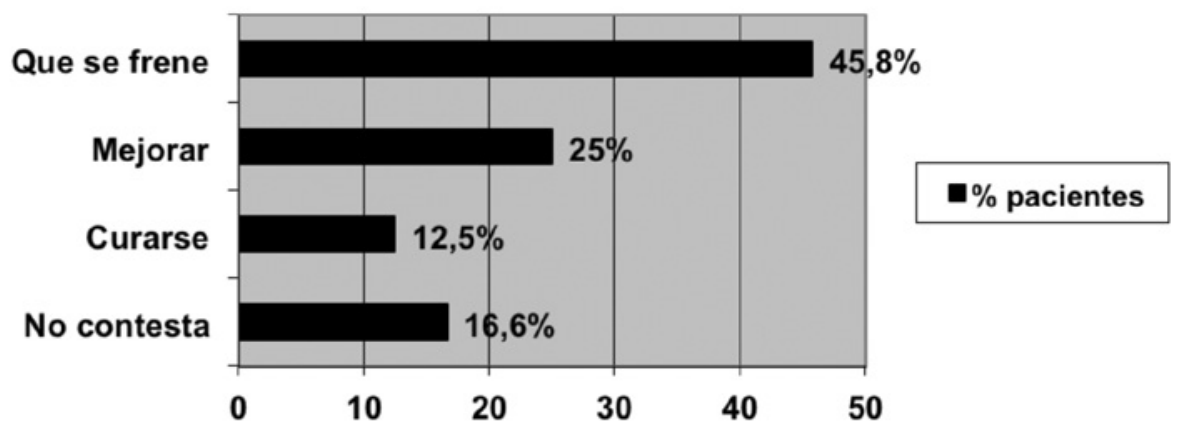


mucho la cabeza" y de hecho la paciente presentaba una carcinomatosis meníngea que se diagnosticó en el transcurso de la enfermedad. Aunque en general, sí que los distinguen y notan como el tratamiento lo que hace es disminuir los síntomas de la enfermedad como el dolor, traducción al castellano: "Yo lo noto porque antes tenía un dolor muy fuerte en la espalda y ahora veo que no tengo dolor, puedes hacer cosas y te encuentras mejor".

\section{Los efectos secundarios}

Algunas pacientes apenas presentan efectos secundarios, o en caso de tenerlos los resuelven por sí mismas "sinceramente lo llevo bien, me canso pero yo sola me animo". Sin embargo, otras presentan muchos efectos secundarios, incluso con las quimioterapias orales tipo capecitabina que a priori pueden parecer menos tóxicas que las endovenosas "eso no es calidad de vida, vivir así no es vivir, no se lo deseo a nadie, no tengo autonomía para nada, úlceras en la boca, grietas en las manos, toda una serie de cosas que componen una lista kilométrica". En cuanto a los tratamientos hormonales también manifiestan algunos efectos secundarios, pero son de una índole más Ilevadera: "es el doble de la menopausia, no tiene nada que ver".

Se les preguntó de qué manera intentaban combatir los efectos secundarios y la mayoría contaban con soluciones que les había dado el médico, conocidos o con iniciativas propias al respecto. Así, las pacientes con quimioterapia oral utilizan cremas de aloe vera, de urea, etc. Algunas pacientes encuentran alivio con el descanso, pero en general utilizan unas estrategias de afrontamiento más activas: "yo leo todo sobre el tema, descarto lo que veo que no es lógico, porque también se escriben barbaridades, intento paliarlos, pero a veces es como darse contra una pared, es hipotecarte la vida, estás las 24 h del día pensando en que te pondrás y haciéndote cosas". Para paliar los efectos secundarios de la hormonoterapia no hay muchos remedios, las pacientes lo soportan y hasta lo asumen con humor, traducción: "no encuentras nada para disminuir las calores, no creo que exista más remedio que ir a tomar el fresco". En algún caso, las pacientes recurren a la homeopatía para contrarrestar los efectos del tratamiento, en este grupo en concreto, únicamente una paciente verbalizó hacer uso de ella.

Respecto a la posibilidad de poder escoger el tratamiento, las pacientes que presentaban respuesta al tratamiento, preferían no cambiarlo, a pesar de que fuera quimioterapia endovenosa: "son los que funcionan"; en 4 casos preferirían más no hacer ningún tratamiento; en 3 casos únicamente zometa (bifosfonatos endovenosos) y en 1 caso radioterapia (RDT); en dos casos reconocen que no saben: "yo ya no sé".

\section{Las exploraciones complementarias}

En cuanto a las exploraciones complementarias, las pacientes acostumbran a considerarlas suficientes y lo dejan a juicio del médico. Reconocen cierto nerviosismo los días previos a la prueba, pero a su vez les da seguridad el saber como están, tienen más sensación de control y más información. A la pregunta si son excesivas, ninguna lo considera así. Respecto a la calidad de vida, aseguran que las pruebas les mejora la calidad de vida porque tienen más información sobre la enfermedad, literal: "¿las pruebas empeorar la calidad de vida? Por supuesto que no, van dirigidas a mejorar mi calidad de vida" y otro ejemplo: "las pruebas complementarias me dan seguridad, prefiero que me hagan controles, las tolero bien".

Sí que en un caso la paciente expresó que las pruebas complementarias no era que fueran excesivas, sino que conside- 
raba que no eran las adecuadas: "yo creo que a veces no son ni excesivas ni suficientes, sino equivocadas. Pienso que hay pruebas que son de más fiabilidad y que equivalen a dos o tres de las normales. Nos someten a más radiación de la cuenta. Sé que hay varios tipos de cáncer, y con un análisis podrían saber a qué tipo pertenece el que tengo y aplicar el tratamiento de una forma más individualizada. Pienso que a estas alturas nos meten a todas en el mismo saco y eso disminuye la eficacia y encarece el tratamiento".

\section{Opiniones sobre aspectos psicosociales}

Apoyo sociofamiliar

De las 24 entrevistas realizadas, en 16 casos $(66,6 \%)$ las pacientes consideran que reciben soporte social y familiar, en 5 casos $(20,8 \%)$ manifiestan no tenerlo y en 3 casos (12,5\%) no solicitarlo.

A la pregunta directa con quién comparte su malestar emocional, las respuestas son generalmente negativas. Según se muestra en la figura 3 un tercio de los pacientes no comparte su malestr con nadie. En muchos casos las pacientes refieren no tener a nadie con quien compartir el malestar, en algunos casos por falta de relaciones sociales, en otros, es porque, a pesar de tener familia (marido e hijos), no se sienten comprendidas: "a pesar de que mis tres hijos se dedican al ramo de la medicina, yo no soy una paciente, soy la madre, no lo quieren ver, se niegan a aceptarlo, no me ayuda que me digan - Jo mamá que estás muy bien!!! Actúan de buena fe para quitarle importancia, pero es que tiene la importancia que tiene, no se le puede ni quitar ni poner. Por eso muchas veces me abstengo de hacer comentarios porque total para lo que me van a decir". De las pacientes que viven en pareja, sólo 2 pacientes $(11,1 \%$ ) dicen compartir su malestar con su marido, en algunos casos porque no quieren agobiar a la familia y en otros porque es la pareja la que adopta una actitud evasiva. De hecho en este grupo se ha dado un caso de abandono por parte del marido cuando la paciente estaba en plena progresión de la enfermedad.

Aspectos de la enfermedad que más le cuesta afrontar

Un aspecto difícil de afrontar y que les preocupa de forma principal, es el entorno, especialmente el cuidado de los hijos, los padres y la relación de pareja. Hay expresiones de las enfermas que describen muy bien esta preocupación: "mi miedo es que mi hijo se quede solo pequeño sin mí, otra paciente expresa: "yo luchaba siempre porque mis padres no se preocuparan y yo creo que esto fue lo que me echó para adelante", y otra paciente "me cuesta asumir que esto me haya pasado

\section{Figura 3. ¿Con quién comparte el malestar?}

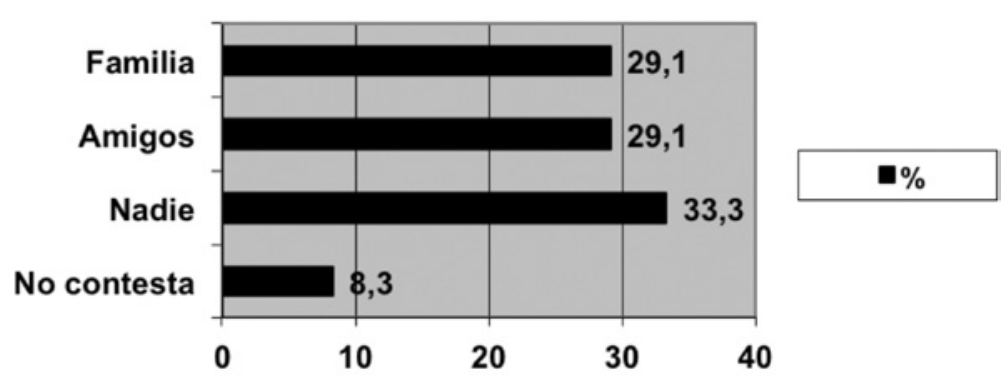


con 40 años, me rompió la vida, ha afectado mi vida sexual, lo único que tenía era mi vida en pareja y hasta esto se me está yendo de las manos, te sientes impotente, eso influye en la vida, no fisiológicamente, sino a nivel afectivo".

Otro aspecto recurrente es no poder llevar la vida de antes, junto a la familia: "al final me acostumbré a saber lo que tenían que hacer, y no les podía impedir que hicieran sus cosas, pero sí que pienso que ha sido muy duro, tanto para mí como para ellos". Y el no poder hacer las tareas cotidianas en parte por el cansancio que comporta la enfermedad y los tratamientos, textual "me canso mucho, me pongo pero no puedo, a veces me enfado, pero la que salgo perdiendo soy yo".

Otro aspecto que les cuesta afrontar, es el miedo a la incertidumbre y a la progresión de la enfermedad, textual: "el no saber por dónde va a salir y esperar, la espera es desesperante". Expresan miedo a los cambios de tratamiento como síntoma de progresión de la enfermedad. Cada cambio de tratamiento traduce el fracaso del anterior. El temor a sentirse sola, especialmente cuando se acerque el final de la enfermedad, también es una preocupación importante.

En menor medida también expresan preocupación por los síntomas físicos. El dolor suele ser un aspecto problemático, en muchos casos se infravalora porque las pacientes minimizan los síntomas: "no soy una persona de comerme la cabeza con estas cosas", en otro caso: "estoy muy ocupada siempre y no tengo tiempo de pensar en tonterías". Otros síntomas referidos son la alopecia, el incremento de peso que hace que no se reconozcan frente al espejo, literal: "Una de las cosas que me costó mucho fue perder el pecho, pero no me costó tanto como verme sin pelo, pero lo peor fue que me puse gorda, por lo menos $15 \mathrm{Kg}$. Y cuando abrí el armario no me cabía nada, y empecé a llorar amar- gamente".

¿Qué cosas/actividades disminuyen su malestar emocional? ¿Qué actividades diarias le ocupan más tiempo? ¿A qué querría dedicar más tiempo?

En general, todas las mujeres utilizan un estilo de afrontamiento activo frente a la enfermedad. Un porcentaje elevado de pacientes encuentran alivio al salir de casa argumentándolo con frases como: "Me gusta salir a pasear con mi perro, si te encierras en ti mismo eso causa malestar. Yo creo que el estado de ánimo influye mucho en la enfermedad, cuando estoy triste y deprimida parece que me duele más todo el cuerpo". Otro comentario muy gráfico es el siguiente: "A mí lo que me ayuda es ir al pueblo, ver la montañas, me hace olvidar la enfermedad".

En general son pacientes muy activas, con ganas de hacer cosas: viajar, caminar, hacer gimnasia, natación, textual: "siempre me busco algo que hacer: leer, escribir, pintar, coser". Algunas prefieren cocinar, hacer manualidades, escuchar música. En general prefieren estar distraídas y no pensar en la enfermedad de base. Alguna paciente incluso refiere querer escribir un libro, textual:"Me gustaría escribir un libro porque tengo tema para hacerlo. Si el físico me acompaña, porque de mente me encuentro como si tuviera 20 años, no me faltan las ganas de hacer cosas, no me acobardo mientras pueda". Ver la figura 4.

Según lo que se observa en la figura 4, la actitud de las pacientes para hacer frente al malestar emocional es pro-activa: distraerse para no pensar en la enfermedad.

Otras pacientes les da seguridad el tener una rutina, por ejemplo una de ellas explica: "paro el despertador, me levanto, me tomo el café, me ducho y salgo a la calle", otra explica "todos los días me levanto, me visto, cojo el coche y me voy con mis amigas al Alcampo, desayunamos, 


\section{Figura 4. ¿Qué cosas disminuyen el malestar emocional?}

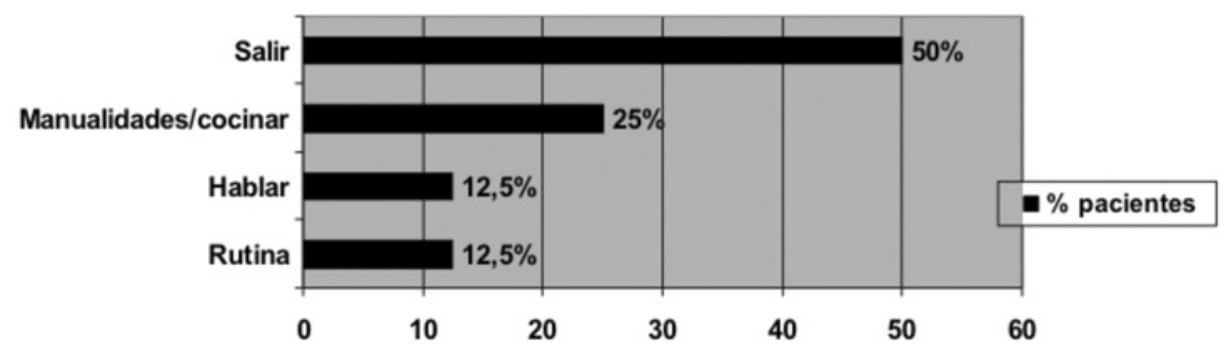

nos despedimos y a hacer faena".

Las tareas domésticas les ocupan mucho tiempo, a pesar de que algunas tienen ayuda externa para hacer las tareas más costosas de la casa, o cuentan con la ayuda del marido para ciertas actividades como comprar o cocinar, pero continúan siendo ellas las que llevan la casa. Expresan la frustración de no poder hacer las cosas de la casa como antes, textual: "Me gustaría hacer la cocina más a fondo, pero no puedo y esto me frustra, me gusta cocinar y me esfuerzo. Lo que me cuesta es planchar, antes lo planchaba todo, hasta los trapos del polvo".

El poder hablar con alguien, estar con gente, también les ayuda. No perder el contacto con los compañeros del trabajo, por ejemplo una maestra comenta "siguen contando conmigo, participo en todo lo que puedo, me apoyan mucho, me quieren". Otra paciente refiere contar con tres compañeras del grupo de terapia "cuando tengo malestar cuento con las del grupo que formamos cuando entramos en la terapia de grupo, porque son personas que me entienden porque están en el mismo columpio que yo". Otra respuesta muy ilustrativa de cómo afrontan la situación es: "salgo a dar una vuelta o llamo a una amiga. Claro que tienes tus momentos de bajón, pero lo hablas, lo comentas, te distraes y ya está".

Las pacientes con hijos pequeños, -pocas-, consideran que su actividad principal y a la que quieren dedicarle más tiempo son los niños, textual: "lo que me ocupa más tiempo son mis hijos", otra paciente expresa: "Mi hijo es lo principal, me gusta ir a Vinaroz con mi hijo, me da energía, es donde esperaba hacer mis planes de futuro". Algunas ya tienen nietos y su ilusión es estar con ellos, textual: "Me gustar estar con mis nietos, me distraen". Aunque no todas tienen el físico para hacerlo debido a los síntomas de la enfermedad o los efectos de los tratamientos, textual: "no puedo salir porque me siento insegura por miedo a la diarrea y no puedo hacer esfuerzos, pero dentro de casa tengo mi vida, mis nietos y me siento bien.

¿Hasta qué punto la enfermedad disminuye su calidad de vida?

A la pregunta si la enfermedad ha disminuido su calidad de vida, es obvio que a todas les ha afectado y cada una lo expresa de una manera u otra, textual: "la enfermedad ha disminuido mi calidad de vida, pues claro". Ver la figura 5.

Un problema muy importante es la limitación física por los síntomas de la enfermedad y los efectos secundarios, textual: "Estoy limitada por los dolores que tengo. Tuve que dejar de trabajar y esto me ha quitado calidad de vida, trabajando te sientes mejor, más independiente, más persona". El cansancio limita la calidad de vida porque no pueden hacer las actividades que hacían antes, han 
tenido que abandonar sus aficiones, sus trabajos, las tareas domésticas dejarlas a cargo de otros y ese hecho las disgusta, les origina ansiedad, disminuye su calidad de vida, textual: "Se que no soy la persona que era antes de la enfermedad, tengo que aceptarlo, tienes cambios de humor, todo depende del estado de ánimo en que te encuentres". Otra paciente explica su limitación por afectación del plexo braquial: "El problema es la mano, como si la tuviera metida en un enchufe". Reconocen estar más limitadas físicamente, textual "Me gustaría caminar pero me canso", otra: "no puedo ir a la piscina porque tengo úlceras en los pies".

Otro problema en este subgrupo de pacientes es el miedo, textual: "Y siempre está el miedo. Cuando coges esta enfermedad, el miedo ya no se va, sabes que lo tienes", y la ansiedad: "la enfermedad te da ansiedad porque lo que hacías antes no lo haces, con la enfermedad todo te afecta mucho más".

Otro aspecto adverso es perder la autonomía tanto por los síntomas de la enfermedad, como por la dependencia del hospital, textual: "nunca te relajas, cada tres semanas estás aquí en el hospital de día, es la realidad pero tú no quieres" y otra paciente expresa: "estoy muy pendiente de los médicos, no tienes esa libertad que tenías antes".

Según la figura 5, el aspecto que más empeora la calidad de vida de las pacientes con cáncer de mama metastático es la pérdida de autonomía.

\section{¿Qué es lo que más le preocupa?}

La preocupación principal de las pacientes son las personas cercanas, de forma preferente los hijos, textual: "Lo que más me preocupa es mi hijo". Y una paciente expresa: "Sigo adelante por mi hijo" y aún más "deseo estar unos años más con mi hija, morirme no me preocupa".

La duración de la respuesta, la incertidumbre sobre la evolución de la enfermedad y el miedo a la progresión lo manifiestan de la siguiente manera: "es una perspectiva diferente del resto de los mortales, van pasando los años y es como si fuera un año menos", "me preocupa saber hasta dónde llegará, hasta cuando responderé al tratamiento" y "Que la enfermedad siga avanzando y que sea pronto, es un pánico horroroso".

A las pacientes también les preocupa el sufrimiento físico, aunque no siempre lo expresan: "Lo único que me preocupa es el dolor. Estoy muy en paz con mi conciencia, con mi manera de ser". Son pacientes que tienden a minimizar los sín-

Figura 5. ¿Qué aspectos de la enfermedad empeoran la calidad de vida?

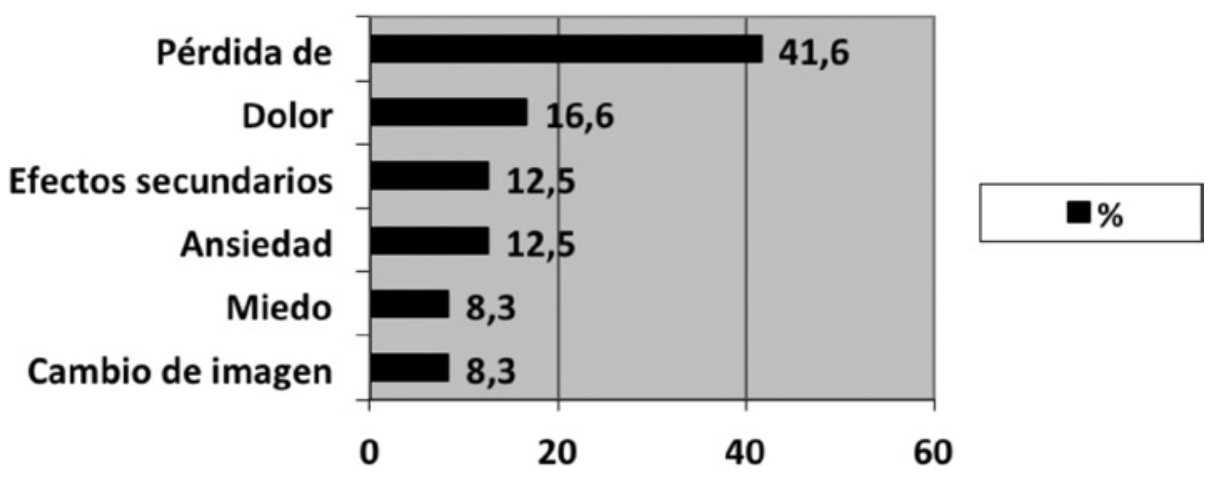




\section{Figura 6. ¿Cuáles son sus preocupaciones?}

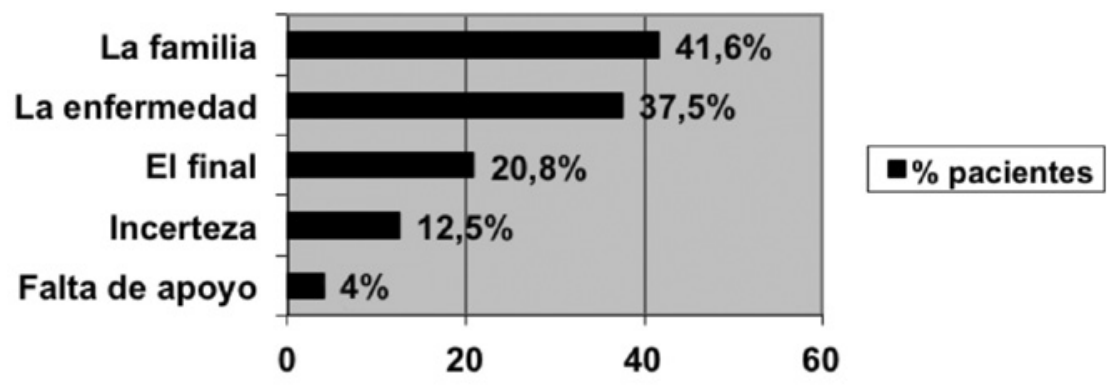

tomas. Ver la figura 6.

Según se observa en la figura 6, la preocupación principal de las pacientes metastásicas es la familia, en especial los hijos.

¿Considera que hay algún aspecto positivo o que ha aprendido alguna cosa de la enfermedad?

En general piensan que la enfermedad no mejora en ningún aspecto su calidad de vida, aunque una proporción importante considera que sí ha aportado algún aspecto positivo (ver figura 7).

Las pacientes con niños pequeños valoran tener más tiempo para dedicarles, textual: "Estar con mi hijo. Antes trabajaba todo el día y prácticamente no lo veía y claro me estaba perdiendo mucha parte de su crecimiento, a raíz de la enfermedad puedo estar con mi hijo, puedo disfrutar de mi hijo y dedicarme a mi hijo".

Entre las pacientes que contestaron abiertamente a esta pregunta, en 11 casos habían aprendido cosas gracias a la enfermedad como ser más considerada con los problemas ajenos, más sensibles a las necesidades de los otros, tener más calidad humana, ser más tolerante.

Sin embargo en 6 casos refieren que la enfermedad no les ha aportado nada, entre otras razones argumentan que ya eran buenas personas antes de caer enfermas.

En 3 casos admiten que ha habido aspectos positivos de crecimiento personal, pero en otros aspectos no. En palabras textuales: "Mi vida ha ido mejorando conforme han pasado los años pero no por la enfermedad, sino por otras cosas. Por una

\section{Figura 7. ¿Considera que la enfermedad aporta algún aspecto positivo?}

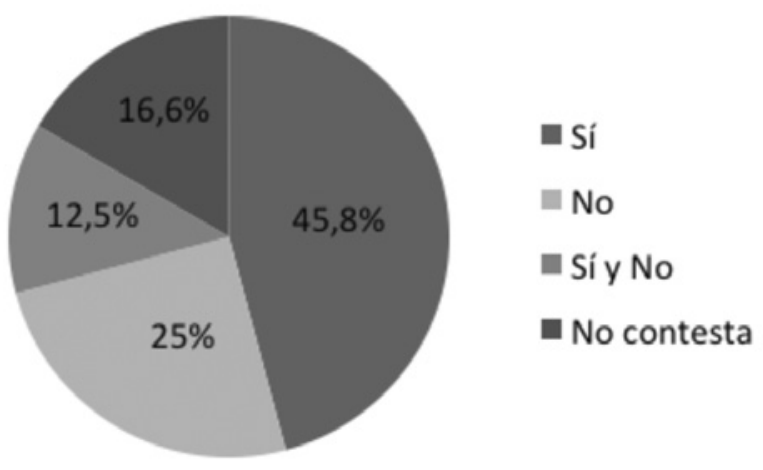


parte sí que me ha ido bien porque he encontrado mis verdaderos amigos, ahí te encuentras a tu familia y a tus amigos que te quieren de verdad".

Como se observa en la figura 7, el $45,8 \%$ de las pacientes entrevistadas consideran que la enfermedad ha aportado algún aspecto positivo en sus vidas.

\section{DISCUSIÓN}

El subgrupo de pacientes con cáncer de mama metastásico es cada vez más numeroso, dado que gracias a la mejoría de los tratamientos viven más años, con todo hay evidencia que el cuidado psicosocial de las pacientes con cáncer de mama metastásico es insuficiente ${ }^{(15)}$.

En una auditoría en un centro australiano de 842 pacientes atendidas, solo el $7 \%$ eran mujeres metastásicas, a pesar de que este grupo representaba el 35\% de los casos de cáncer de mama de dicho hospital ${ }^{(16)}$. De nuestro estudio únicamente 7 pacientes de 24 estaban recibiendo apoyo psico-social en el momento de la entrevista (29\%).

La escasa bibliografía existente acerca de las enfermas metastásicas, muestra que son pacientes que presentan síntomas no resueltos como el dolor, cansancio, disnea, insomnio y pérdida de apetito ${ }^{(17,18)}$ y altos niveles de malestar emocional, especialmente las pacientes con dolor ${ }^{(19)}$ y necesidades cotidianas no cubiertas como el trabajo doméstico, asistencia financiera y ayuda para el transporte ${ }^{(20)}$. En términos de calidad de vida, más de una cuarta parte de las pacientes metastásicas expresan un peor estado de salud y más de la mitad expresan estar fatigadas; el dolor y el insomnio también las afecta, aunque lo expresan en menor medida.

Debido a la naturaleza subjetiva de la calidad de vida, el impacto emocional del diagnóstico de enfermedad metastásica no puede ser recogido únicamente utili- zando escalas de calidad de vida predeterminadas. Los instrumentos de calidad de vida existentes no pueden recoger la individualidad de cada una de las pacientes metastásicas.

El término "necesidades no atendidas", en inglés "the unmet needs" se define como la necesidad de alguna intervención o recurso que es necesario, deseable o útil para conseguir el bienestar ${ }^{(21)}$. Intentar descubrir cuáles son las necesidades no atendidas de las pacientes con cáncer de mama metastásicas puede completar la información obtenida a través de los cuestionarios de calidad de vida existentes.

Entre la población anglosajona, las necesidades no atendidas más frecuentes en las pacientes con cáncer de mama avanzado son la falta de información médica, especialmente sobre tratamientos experimentales, sobre el apoyo psicológico y social y las dificultades físicas para las tareas diarias $^{(22)}$.

Las necesidades también cambian a lo largo de la evolución de la enfermedad. En un estudio realizado con mujeres con cáncer de mama avanzado pero diagnóstico reciente ${ }^{(23)}$, los resultados mostraron que las preocupaciones más nombradas fueron: las dificultades de comunicación con los médicos $(61,2 \%)$; el retraso percibido en el diagnóstico de la recidiva (41,5\%); el impacto emocional, principalmente la incertidumbre $(32,8 \%)$ y la pérdida de independencia (31,3\%); preocupaciones relacionadas con el impacto de la enfermedad en sus familias $(35,8 \%)$, principalmente en los hijos $(55,2 \%)$ y preguntas sobre el porqué de la progresión de la enfermedad.

En comparación respecto a este grupo de pacientes, las pacientes con cáncer de mama avanzado presentan mayores niveles de necesidades no atendidas en el ámbito psicosocial y en las tareas domésticas ${ }^{(24)}$. En nuestro estudio, que se caracteriza por incluir pacientes metastásicas de larga evolución, los síntomas de malestar emo- 
cional son inferiores a lo esperado ${ }^{(25)}$. Probablemente la adaptación en el tiempo de estas pacientes, hace que sean capaces de controlar los niveles de ansiedad y miedo que una enfermedad incurable ofrece de entrada. Las pacientes continúan en su rol de piedra angular de la familia y su preocupación principal no es su enfermedad, sino el cuidado de los suyos, especialmente de los hijos. En nuestro estudio la preocupación principal son las personas cercanas en un $41.6 \%$, seguido de preocupaciones por la evolución de la enfermedad en un $37,5 \%$ y el miedo a la incertidumbre sobre el futuro o el final de la vida en un 12,5 y $20,8 \%$, respectivamente.

En un estudio australiano ${ }^{(26)}$ con 172 pacientes metastásicas, con características clínicas semejantes a nuestro grupo, se evaluó la calidad de vida utilizando la escala de calidad de vida de la EORTC QLQ-C30 y un cuestionario sobre necesidades para el tratamiento de apoyo (SNCQ: Supportive Care Needs Questionnaire) para complementar el análisis del cuestionario de la EORTC. En su estudio, los dos factores considerados como las necesidades no atendidas de mayor importancia fueron: la preocupación por los demás (familiares cercanos) y ser informadas acerca de estrategias que ellas puedan aplicarse para sentirse mejor. Estos resultados son comparables con los de nuestro grupo de pacientes.

Si comparamos el estudio de SansonFisher et al. ${ }^{(21)}$ en población oncológica heterogénea con el de Aranda et al.(26), se observa que si bien las necesidades no atendidas de las pacientes con cáncer de mama metastásico eran similares al resto de pacientes con otras patologías neoplásicas, se diferenciaban en el orden de prioridades. Mientras que en el grupo heterogéneo de pacientes, las tres preocupaciones principales eran: "miedo a la progresión del cáncer", "miedo a la recidiva" y por último "preocupación acerca de las personas próximas"; en las pacientes con cáncer de mama metastásico, el orden de los valores se invierte, estando en primer lugar, "la preocupación por las personas próximas"; en segundo lugar, "ser informado acerca de las estrategias para sentirse mejor" y por último, "la incertidumbre sobre el futuro".

Cabe destacar que la necesidad de ser informadas que encontramos en los estudios anglosajones no es una preocupación tan evidente en nuestras pacientes. Este hecho puede deberse a diferencias sociales entre la cultura anglosajona y la nuestra mediterránea. Por otra parte, el paciente ha ido asumiendo un papel más activo frente a su enfermedad, buscando más información y solicitando aclarar sus dudas. De todos modos, las pacientes deben de ser informadas correctamente y los profesionales atender las dudas que se les plantean tanto en cuanto al diagnóstico y pronóstico como en referencia a los tratamientos aprobados y en estudio, así como de los efectos secundarios y la mejor manera de paliarlos.

Ryan et al. ${ }^{(27)}$, en su estudio enfatiza que en la ausencia de un mecanismo formal para obtener información sobre las necesidades reales de la enfermas, los profesionales que las atienden deberían preguntar directamente a las pacientes sobre su calidad de vida y sus necesidades no atendidas. El hecho de utilizar preguntas abiertas, escuchar de forma activa, responder a las preguntas de una manera empática y aclarando dudas de las pacientes, son todas ellas estrategias que ayudan a entender las preocupaciones reales de nuestras pacientes. Esta estrategia es parecida a la que hemos querido utilizar en nuestro trabajo. Nuestra propuesta de preguntas abiertas no pretende dar un formulario con nuestras preguntas exactas, sino dar la oportunidad a las pacientes de que puedan expresarse libremente sobre la preocupaciones que realmente les atañen, que no siempre tienen que estar relacionadas con la enfermedad y que condicionan realmente su 
calidad de vida. En concreto, los datos más relevantes en nuestro estudio sobre las preguntas abiertas son los datos referentes al apoyo psicosocial, a los aspectos que más les cuesta afrontar, que actividades mejoran o empeoran su calidad de vida, cuáles son sus preocupaciones y en qué medida la enfermedad representa algún aspecto positivo en su calidad de vida.

Las preguntas referentes a los aspectos médicos también han revelado datos que los profesionales no somos tan conscientes. Por ejemplo, respecto al beneficio obtenido por el tratamiento más de la mitad reconocen que ha mejorado la calidad de vida, con todo un $20 \%$ declara no encontrar mejoría y un $16 \%$ sólo en algunas aspectos. Más interesante es ver lo que las pacientes esperan del tratamiento, la mayoría se conforman en que la enfermedad se detenga, una cuarta parte espera mejorar y sólo algún caso aspira a curarse. Se desprende de sus respuestas que las pacientes saben que se enfrentan a una enfermedad metastásica y por lo tanto incurable. Respecto a los efectos secundarios, nos ha sorprendido que los tratamientos con quimioterapia oral no sean tan inocuos como parecen a priori. En cambio los efectos secundarios de la quimioterapia endovenosa los sobrellevan mejor, probablemente porque los asocian a mayor respuesta ${ }^{(28,29)}$. Los efectos secundarios de la hormonoterapia son más conocidos y no distan de los publicados ${ }^{(30)}$. Respecto a las exploraciones complementarias, si bien les provocan cierto nerviosismo, prefieren tener un buen control sobre su enfermedad. El número y tipo de exploraciones complementarias lo dejan a criterio del profesional.

Uno de los datos más relevantes de nuestro estudio ha sido el insuficiente apoyo psicosocial percibido por las pacientes. A la hora de compartir su malestar emocional, un tercio de nuestras pacientes no lo comparten con nadie. En la mayoría de los casos, son las pacientes que evitan preocupar a la familia, con todo, en ocasiones la familia también puede adoptar una actitud de negación. Un estudio sobre el enfoque diático en cáncer de mama metastásico, demuestra que para asegurar un buen apoyo mutuo de la pareja frente debe haber comunicación, apoyo positivo y apoyo mutuo ${ }^{(31)}$. Muchas parejas están estresadas y no ofrecen el apoyo necesario, adoptan actitudes de evitación, de crítica e incluso minimizan el problema ${ }^{(32,33)}$.

Por último, entre los ítems que nuestras pacientes relacionan en mayor medida con la pérdida de calidad de vida son la pérdida de autonomía, tanto por la limitación de los síntomas de la enfermedad como el dolor, la disnea, como por los efectos secundarios de los tratamientos, o por el simple hecho de estar pendientes continuamente de los horarios de las visitas y de los tratamientos.

Al preguntarles si la enfermedad ha aportado algún aspecto positivo en su calidad de vida, menos de la mitad afirman haber aprendido algo de la enfermedad. Sí que reconocen que gracias a la enfermedad han descubierto las personas que realmente se preocupan por ellas, las ha hecho cambiar en la actitud hacia los demás volviéndose más consideradas, más sensibles a los problemas ajenos. Con todo, algunas de las pacientes reconocen que ya eran así antes de la enfermedad. Otro valor añadido, es que a las pacientes jóvenes les ha permitido estar más cerca de sus hijos, al menos el tiempo en que han estado con vida.

Teniendo en cuenta las dificultades inherentes para reclutar enfermas metastásicas, el interés del presente estudio es que va dirigido específicamente a evaluar los niveles de calidad de vida en pacientes con cáncer de mama metastásico. Un estudio de estas características en población catalana no se ha publicado hasta la fecha en nuestro conocimiento. Por este motivo, nos parece interesante describir la percepción de cali- 
dad de vida de nuestras propias pacientes, que si bien coincide con la de la población anglosajona, es ligeramente diferente.

El hecho más destacable de este subgrupo de pacientes con cáncer de mama de larga evolución es lo bien adaptadas que están a esta situación crónica pero incurable, lo admirable es su actitud delante de la vida que les ha tocado vivir; preocupándose más por los demás que por ellas mismas; la manera en que minimizan los síntomas para no preocupar a los demás y el poco uso que hacen de los recursos sanitarios teniendo en cuenta su delicada situación de salud.

Los estudios revisados destacan la importancia de proporcionar información y apoyo a las mujeres afectadas. Valorar las necesidades de las pacientes metastásicas y su posterior derivación al profesional adecuado, en caso necesario, debería ser una parte integral de la valoración de la salud ${ }^{(34,39)}$.

Mejorando la calidad de vida y atendiendo las necesidades de nuestras pacientes metastásicas, no solo repercute en nuestras pacientes y familiares, sino que reduce ostensiblemente el gasto sanitario. Se sabe que las pacientes con necesidades psicosociales no atendidas hacen un mayor uso de los servicios sanitarios, presentan estancias hospitalarias más prolongadas y encarecen más la atención médica que los pacientes no deprimidos ${ }^{(40,41)}$. Por lo tanto, añade un punto de atención más la preocupación por la calidad de vida de estas pacientes.

Ante esta enfermedad incurable, mejorar la calidad de vida y resolver las necesidades no atendidas de nuestras pacientes con cáncer de mama metastásico debería ser el objetivo principal de nuestra labor como profesionales de la salud.

\section{Agradecimientos}

A todas y cada una de las pacientes que han participado en el estudio y a aquellas que por causas ajenas a su voluntad no lo han podido hacer, gracias por su lección de vida diaria. Agradecer también a la Asociación Española contra el Cáncer de Barcelona y al servicio de epidemiología de l'Institut Català d'Oncologia de l'Hospitalet por la prestación de sus instalaciones y por el soporte técnico, respectivamente.

\section{REFERENCIAS BIBLIOGRÁFICAS}

1. Stewart BW, Kleihues P. World Cancer Report. International Agency for Research on Cancer, Lyon, France, 2003.

2. Izquierdo A, Gispert R, Saladie F, Espinàs JA. Análisis de la incidencia, la supervivencia y la mortalidad según las principales localizaciones tumorales, 1985-2019: cáncer de mama. Med Clin (Barc) 2008;131(supl.1):50-2.

3. Hagedoorn M, Sanderman R, Bolks HN, Tuinstra J, Coyne JC. Distress in couples coping with cancer: A meta-analysis and critical review of role and gender effects. Psychol Bull 2008;134:1-30. Doi: 10.1037/0033-2909.134.1.1.

4. Butler L, Koopman C, Cordova M, Garlan R, DiMlceli S, Spiegel D. Psychological distress and pain significantly increase before death in metastatic breast cancer patients. Psychosom Med 2003;65:416-26. Doi: 10.1097/01.PSY.0000041472.77692. C6

5. Acharya J, Sethugavalar B, Mansell J, Dodwell D, Adlard J. Changes in metastatic breast cancer management over 5 years - costs and outcomes. National Cancer Research Institute, Birmingham: NCRI, 227, 2008.

6. DeVita VT, Lawrence TS, Rosenberg SA, editors. DeVita, Hellman, and Rosenberg's Cancer: Principles \& Practice of Oncology, 9th Edition. Philadelphia: Lippincott Williams \& Wilkins, 2011.

7. Brescia FJ, Adler D, Gray G, Ryan M, 
Cimino J, Mantani R. Hospitalized advanced cancer patients: A profile. J Pain Symptom Manage 1990;5:221-7. Doi: 10.1016/0885-3924(90)90015-C.

8. Kramer JA, Curran D, Piccart M, de Haes CJM, Bruning P, Klijn J, et al. Identification and interpretation of clinical and quality of life prognostic factors for survival and response to treatment in first-line chemotherapy in advanced breast cancer. E J Cancer 2000;36:1498-506. Doi: 10.1016/S0959-8049(00)00144-1.

9. Cella D, Tulsky DS, Gray G, Sarafian B, Linn $E$, Bonomi $A$, et al. The functional assessment of cancer therapy (FACT) scale: Development and validation of the general measure. J Clin Oncol 1993;11:570-9.

10. EORTC Quality of Life Group. EORTC Quality of Life Questionnaire: EORTC QLQ-C30. Qual Life Res 1993;2:287-95.

11. Fayers P, Aaronson NK, Bjordal K, Curran $D$ and Groenvold $M$ on behalf of the EORTC Quality of Life Study Group. EORTC QLQ-C30 Scoring Manual (Third edition). Brussels, EORTC Quality of Life Group, 2001, 86 pp.www.eortc.be/home/qol.

12. EORTC Quality of Life Questionnaire module for Breast Cancer Patients: EORTC QLQ-BR23. J Clin Oncol 1996;14:2756-68.

13. Font A. Valoración de la calidad de vida en pacientes de cáncer. Tesis doctoral. Universidad Autónoma de Barcelona. Bellaterra: Barcelona, 1988.

14. Font A. Cáncer y calidad de vida. An Psicol 1994;61:41-50.

15. Parle M, Gallagher J, Gray C, Akers G, Liebert B. From evidence to practice: Factors affecting the specialist breast nurse's detection of psychological morbidity in women with breast cancer. Psychooncology 2001;10:503-10. Doi: 10.1002/pon.541.

16. Aranda S, Milne D, Osmond F. A Breast Care Nurse Database Evaluation Report. Victorian Centre for Nursing Practice Research, Melbourne, Australia, 2002.

17. Vachon ML, Kristjanson L, Higginson I.
Psychosocial issues in palliative care: The patient, the family, and the process and outcome of care. J Pain Symptom Manage 1995;10:142-50. Doi: 10.1016/08853924(94)00076-W.

18. Wong RK, Franssen E, Szumacher E, Connolly R, Evans M, Page B, et al. What do patients living with advanced cancer and their carers want to know? A needs assessment. Support Care Cancer 2002;10:408-15. Doi: 10.1007/s00520-002-0354-3.

19. Stromgren AS, Groenvold M, Sorensen A, Andersen L. Symptom recognition in advanced cancer. A comparison of nursing records against patient self-rating. Acta Anaesthesiol Scand 2001;45:1080-5. Doi: 10.1034/j.1399-6576.2001.450905.x.

20. Davies M, Sque M. Living on the outside looking in: A theory of living with advanced breast cancer. Int J Palliative Nurs 2002;8:583-90. Doi: 10.12968/ ijpn.2002.8.12.10975

21. Sanson-Fisher R, Girgis A, Boyes A, Bonevski $B$, Burton L, Cook P. The unmet supportive care needs of patients with cancer. Cancer 2000;88:226-37. Doi: 0.1002/(SICI)1097$0142(20000101) 88: 1 \% 3 C 226:$ AIDCNCR30\%3E3.3.CO;2-G

22. McLachlan S, Allenby A, Matthews J, WirthA, Kissane D, Bishop M, et al. Randomized trial of co-ordinated psychosocial interventions based on patient self-assessments versus standard care to improve the psychosocial functioning of patients with cancer. J Clin Oncol 2001;19:4117-25.

23. Turner J, Kelly B, Swanson C, Allison R, Wetzig N. Psychosocial impact of newly diagnosed advanced breast cancer. Psychooncology 2005;14:396-407. Doi: 10.1002/pon.856.

24. Foot G, Sanson-Fisher R. Measuring the unmet needs of people living with cancer. Cancer Forum 1995;19:131-5.

25. Lidgren $M$, Wilking $N$, Jönsson $B$, Rehnberg C. Health related quality of life in different states of breast cancer. Qual Life Res 2007;16:1073-81. Doi: 10.1007/ 
s11136-007-9202-8.

26. Aranda $S$, Schofield $P$, Weih $L$, Yates $P$, Milne D, Faulkner R, et al. Mapping the quality of life and unmet needs of urban women with metastatic breast cancer. Eur J Cancer Care 2005;14:211-22. Doi: 10.1111/j.1365-2354.2005.00541.x

27. Ryan H, Schofield PE, Cockburn J, Butow $P$, Girgis A, Tattersall $M$, et al. How to recognise and manage psychological distress in cancer patients. Eur J Cancer Care 2005;14:7-15. Doi: 10.1111/j.13652354.2005.00482.x.

28. Karamouzis MV, loannidis G, Rigados G. Quality of life in metastatic breast cancer patients under chemotherapy or supportive care: A single institution comparative study. Eur J Cancer Care 2007;16:433-8. Doi: 10.1111/j.1365-2354.2006.00771.x.

29. Moinpour CM, Wu J, Donaldson GW, Liepa A, Melemed A, O'Shaughnessy J, et al. et al. Gemcitabine plus paclitaxel versus paclitaxel as first-line treatment for anthracycline pre-treated metastatic breast cancer: Quality-of-life and pain palliation results from the global phase III study. J Clin Oncol 2004;22(14s):621.

30. Whelan TJ, Goss PE, Ingle JN, Pater JL, Tu D, Pritchard K, et al. Assessment of quality of life in MA.17: A randomized, placebo-controlled trial of letrozole after 5 years of tamoxifen in postmenopausal women. J Clin Oncol 2005;23:6931-40. Doi: 10.1200/JCO.2005.11.181.

31. Bard H, Carmack C, Kashy DA, Cristofanilli M. Dyadic coping in metastatic breast cancer. Health Psychol 2010;29:169-80. Doi: 10.1037/a0018165.

32. Vilhauer RP. A qualitative study of the experiences of women with metastatic breast cancer. Palliat Support Care 2008;6:249-58. Doi: 10.1017/ S1478951508000382.

33. Hasson-Ohayon I, Goldzweig G, Braun M, Galinsky D. Women with advanced breast cancer and their spouses: Diversity of support and psychological distress. Psychooncology 2010;19:1195-204. Doi: 10.1002/pon.1678

34. Akechi T, Okuyama T, Endo C, Sagawa R, Uchida M, Nakaguchi T, et al. Patient's perceived need and psychological distress and/or quality of life in ambulatory breast cancer patients in Japan. Psychooncology 2011;20:497-505. Doi: 10.1002/pon.1757.

35. Anderson WG, Alexander SC, Rodríguez $\mathrm{KL}$, Jeffreys AM, Olsen MK, Pollak KI et al. "What concerns me is..." Expression of emotion by advanced cancer patients during outpatient visits. Support Care Cancer 2008;16:803-11. Doi: 10.1007/ s00520-007-0350-8.

36. Kenne Sarenmalm E, Öhlén J, Odén A, Gaston-Johansson F. Experience and predictors of symptoms, distress and health-related quality of life over time in postmenopausal women with recurrent breast cancer. Psychooncology 2008;17:497-505. Doi: 10.1002/pon.1258.

37. Mayer M. Living with advanced breast cancer: Challenges and opportunities. Breast 2011;20:S12-S13. Doi:10.1016/j. breast.2011.08.004

38. Reed E, Wheeler D. Five ways we can do better for patients with metastatic disease. European Oncology Nursing Society Newsletter 2009;12-5.

39. Uchida M, Akechi T, Okuyama T, Sagawa R, Nakaguchi T, Endo C, et al. Patients' supportive care needs and psychological distress in advanced breast cancer patients in Japan. Jpn J Clin Oncol 2011;41:530-6. Doi: 10.1093/jjco/hyq230.

40. Fulop G, Strain JJ, Vita J. Impact of psychiatric comorbidity on length of hospital stay for medical/surgical patients: A preliminary report. Am J Psychiat 1987;144:878-82.

41. Simon GE, VonKorff M, Barlow W. Health care costs of primary care patients with recognized depression. Arch Gen Psychiat 1995;52:850-6. 
